LAWRENCE LIVERMORE N A T IO N A L LABORATORY

\title{
Variation in Particle Pumping Due to Changes in Topology in High Performance DIII-D Plasmas
}

T.W. Petrie, N.H. Brooks, M.E. Fenstermacher, C.M. Greenfield, M. Groth, A.W. Hyatt, A.W. Leonard, M.A. Mahdavi, G.D. Porter, M.E. Rensink, M.J. Schaffer, M.R. Wade, J.G. Watkins, N.S. Wolf

December 6, 2004

16th International Conference on Plasma Surface Interactions Portland, ME, United States May 24, 2004 through May 28, 2004 
This document was prepared as an account of work sponsored by an agency of the United States Government. Neither the United States Government nor the University of California nor any of their employees, makes any warranty, express or implied, or assumes any legal liability or responsibility for the accuracy, completeness, or usefulness of any information, apparatus, product, or process disclosed, or represents that its use would not infringe privately owned rights. Reference herein to any specific commercial product, process, or service by trade name, trademark, manufacturer, or otherwise, does not necessarily constitute or imply its endorsement, recommendation, or favoring by the United States Government or the University of California. The views and opinions of authors expressed herein do not necessarily state or reflect those of the United States Government or the University of California, and shall not be used for advertising or product endorsement purposes. 


\title{
Variation in particle pumping due to changes in topology in high performance
}

\section{DIII-D plasmas}

T.W. Petrie ${ }^{\mathrm{a}}$, N.H. Brooks ${ }^{\mathrm{a}}$, M.E. Fenstermacher ${ }^{\mathrm{b}}$, C.M. Greenfield $^{\mathrm{a}}$, M. Groth ${ }^{\mathrm{b}}$, A.W. Hyatt ${ }^{\mathrm{a}}$,

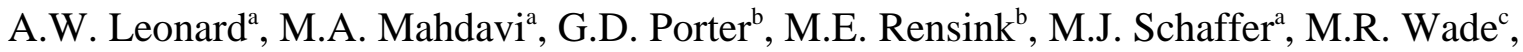
J.G. Watkins ${ }^{\mathrm{d}}$, N.S. Wolf ${ }^{\mathrm{b}}$, and The DIII-D Team

${ }^{a}$ General Atomics, P.O. Box 85608, San Diego, California 92186-5608, USA

${ }^{b}$ Lawrence Livermore National Laboratory, Livermore, California 94550, USA

${ }^{c}$ Oak Ridge National Laboratory, Oak Ridge, Tennessee, USA

${ }^{d}$ Sandia National Laboratories, Albuquerque, New Mexico 87185-1129, USA

\begin{abstract}
Recent experiments on DIII-D point to the importance of two factors in determining the rate at which deuterium particles can be pumped at the divertor target(s): (1) the divertor magnetic balance, i.e., the degree to which the divertor topology is single-null (SN) or double-null (DN), and (2) the direction of the of $B \times \nabla B$ ion drift with respect to the $\mathrm{X}$-point(s). Changes in divertor magnetic balance near the $\mathrm{DN}$ shape have a much stronger effect on the pumping rate at the inner divertor target(s) than on the pumping rate at the outer divertor target(s). The behavior in the particle pumping observed at the inner and outer divertor target(s) in the DN and near-DN shapes suggests a redistribution of particles that would be expected in the presence of $B \times \nabla B$ and $E \times B$ ion particle drifts in the scrapeoff layer (SOL) and divertor(s).
\end{abstract}


JNM keywords: Experimental Techniques-E0500, Plasma-Materials Interaction - P0500,

Plasma Properties - P0600, Theory and Modeling - T0100

PSI-16 keywords: Divertor pumping, Divertor asymmetry, Magnetic topology, Particle drifts,

Recycling

PACS: $52.40 . H f$, 52.55.Fa, 52.70.-m

*Corresponding author address: General Atomics, P.O. Box 85608, San Diego, CA 92186. USA

*Corresponding author e-mail: petrie@fusion.gat.com

Presenting author address: General Atomics, P.O. Box 85608, San Diego, CA 92186. USA

Presenting author e-mail: petrie@fusion.gat.com 


\section{Introduction}

Previous studies of non-actively pumped DIII-D discharges showed that small variations in the magnetic balance near the double-null (DN) configuration can have sizable effects on plasma behavior in the scrapeoff layer (SOL) and in the divertor. The magnetic balance affects the poloidal distributions at the divertor targets of heat and particle fluxes $[1,2]$, the edge localized mode (ELM)-driven particle pulses [3], and the plasma recycling [4]. In addition, ion particle drifts, such as $B \times \nabla B$ and $E \times B$ drifts [5,6], also are thought to contribute to these observed poloidal "asymmetries" $[7,8]$.

In this paper, we present the results of recent experiments in DIII-D, which focus on how active particle pumping is affected by changes in the magnetic balance and in the direction of the toroidal magnetic field $B_{\mathrm{T}}$. We describe our experimental setup in Sec. II, present our results in Sec. III, and discuss these results in Sec. IV.

\section{Experimental setup}

Three examples of the poloidal cross-sections used in this study are shown in Fig. 1(a) unbalanced upper DN, (b) balanced DN, and (c) unbalanced lower DN. In this paper, we refer to the shapes in Fig. 1(a) and 1(c) as "upper SN" and "lower SN", respectively. These plasmas exhibit Type-1 ELMing [9] and have good energy confinement, i.e., $\tau_{\mathrm{E}} / \tau_{\mathrm{E} 89 \mathrm{P}}=(2.1-2.5)$, where $\tau_{\mathrm{E} 89 \mathrm{P}}$ is the 1989 L-mode energy confinement scaling [10]. Repeat shots were taken until the graphite tiles that protect the interior vessel were conditioned and reproducibility of shots was demonstrated.

In-vessel pumping was done from two poloidal locations [Fig. 1(a)]. One of these pumps is located inside the "dome" plenum and exhausted particles at the upper inner divertor target. The second pump is located under the "baffle" plenum and exhausted particles at the upper outer 
divertor target. The locations of the divertor legs were chosen to maximize the respective pumping rates. The rate at which particles were exhausted by the dome pump $\Gamma_{\mathrm{DOME}}$ (or baffle pump $\left.\Gamma_{\mathrm{BAF}}\right)$ was calculated from the product of the neutral pressure measured in the dome plenum (or baffle plenum) and the measured pumping speed $S$. Representative pumping speeds were $\approx 15 \mathrm{~m}^{3} / \mathrm{s}$ for the dome cryopump and $\approx 35 \mathrm{~m}^{3} / \mathrm{s}$ for the upper baffle cryopump. Analysis indicates that before a neutral entering the plenum reaches the neutral pressure gauge, the deuterium has been thermalized by multiple collisions with the plenum structure and is in molecular form.

To quantify the divertor magnetic balance, we define the quantity $d R \operatorname{sep}$ as $\left(R_{\mathrm{L}}-R_{\mathrm{U}}\right)$, where $R_{\mathrm{L}}$ is the radius of the lower divertor separatrix flux surface at the outer midplane and $R_{\mathrm{U}}$ is the radius of the upper divertor separatrix flux at the outer midplane. For example, $R_{\mathrm{U}}<R_{\mathrm{L}}$ and $d$ Rsep $=+1.2 \mathrm{~cm}$ for the upper SN case shown in Fig. 1(a). 


\section{Experimental results}

\subsection{Dependence of $\Gamma_{\mathrm{DOME}} / \Gamma_{\mathrm{BAF}}$ on magnetic balance}

The ratio of inner to outer pumping rates, $\Gamma_{\mathrm{DOME}} / \Gamma_{\mathrm{BAF}}$, decreased steadily, as the magnetic balance changed from an upper SN to a lower SN (Fig. 2). This decrease was observed for both $B \times \nabla B$ ion particle drift directions (i.e., toward the upper divertor and away from the upper divertor). In either $B \times \nabla B$ ion drift direction, the relative importance of $\Gamma_{\mathrm{DOME}}$ to $\Gamma_{\mathrm{BAF}}$ decreased by roughly a factor of 2 , for $d R s e p=(+1 \mathrm{~cm} \rightarrow 0 \mathrm{~cm})$, and decreased further as the plasma shape became a lower SN (i.e., dRsep <0).

\subsection{Dependence of $\Gamma_{\mathrm{DOME}} / \Gamma_{\mathrm{BAF}}$ on the $B \times \nabla B$ ion particle drift direction}

The ratio $\Gamma_{\mathrm{DOME}} / \Gamma_{\mathrm{BAF}}$ was significantly different for cases with opposite $B \times \nabla B$ ion drift directions but with the same $d R s e p$. In the balanced DN case of Fig. 2, $\Gamma_{\mathrm{BAF}}$ is approximately a factor of five greater than $\Gamma_{\mathrm{DOME}}$ when the $B \times \nabla B$ ion drift is directed away from the $\mathrm{X}$-point in the upper divertor. However, $\Gamma_{\mathrm{BAF}}$ is only one third higher than $\Gamma_{\mathrm{DOME}}$ when the $B \times \nabla B$ ion drift is directed toward the $\mathrm{X}$-point in the upper divertor.

\subsection{Dependence of $\Gamma_{\mathrm{DOME}}$ and $\Gamma_{\mathrm{BAF}}$ on dRsep}

A particle balance calculation shows how $\Gamma_{\mathrm{DOME}}$ and $\Gamma_{\mathrm{BAF}}$ depend individually on $d R$ sep:

$$
0=\Gamma_{\mathrm{INJ}}-\Gamma_{\mathrm{DOME}}-\Gamma_{\mathrm{BAF}}-\Gamma_{\mathrm{WALL}}-d N_{\mathrm{c}} / d t
$$

where $\left(\Gamma_{\mathrm{INJ}}\right)$ is the neutral beam fueling rate, $\left(d N_{\mathrm{c}} / d t\right)$ the rate that particles enter the plasma system (if positive), and ( $\left.\Gamma_{\text {WALL }}\right)$ the rate that particles are lost to the graphite tiles protecting the vessel walls, including the lower divertor (if positive). For the cases where the $B \times \nabla B$ ion 
drift is toward the upper divertor, Fig. 3 shows that $\Gamma_{\text {DOME }}$ is clearly sensitive to small changes in magnetic balance near DN. Between dRsep $=0.0 \mathrm{~cm}$ and $+1.2 \mathrm{~cm}$, however, $\Gamma_{\mathrm{BAF}}$ is insensitive to change in $d R s e p$.

As the magnetic balance shifts from an upper $\mathrm{SN}$ to a balanced $\mathrm{DN}$, the graphite tiles that protect the vessel walls appear to "pump" an increasingly higher fraction of the plasma exhaust in order to maintain the pedestal density in "steady state." For $\Gamma_{\mathrm{BAF}} \approx+1.2 \mathrm{~cm}$ in Fig. 3, $\Gamma_{\mathrm{WALL}}$ is roughly one-third of the combined dome and baffle pump rates $\left(\Gamma_{\mathrm{DOME}}+\Gamma_{\mathrm{BAF}}\right)$, so that in a well-conditioned vessel "steady state" (upper) SN discharges could likely be maintained over several seconds under these conditions. Figure 3 also suggests that long-pulse operation in DN under the same density and fueling conditions would require stronger "wall pumping".

\subsection{Dependence of $\Gamma_{\mathrm{DOME}}$ and $\Gamma_{\mathrm{BAF}}$ on recycling activity}

Changes in recycling activity in front of the entrance to the plenum is an indicator of changes occurring in the particle exhaust rate. Figure 4 shows that for both (a) dome and (b) baffle cases, the number of particles exhausted by either the dome or baffle pumps is approximately proportional to the recycling taking place adjacent to their respective pump apertures, e.g., $\Gamma_{\mathrm{DOME}} \propto\left(\Phi_{\mathrm{DOME}}\right)^{1.07}$, where the recycling $\Phi_{\mathrm{DOME}}$ is characterized by the intensity of the $D_{\alpha}$ light radiating from the front of the entrance to the dome plenum.

\section{Discussion and conclusion}

The sensitivity of $\Gamma_{\mathrm{BAF}}$ to changes in $d R s e p$ near DN is much less than that of $\Gamma_{\mathrm{DOME}}$. This result is largely due to the following. We estimate that the number of particles entering the SOL from the low $B_{\mathrm{T}}$-field side of the core plasma is 3-4 times higher than the number that enter from the high $B_{\mathrm{T}}$-field side. This imbalance is due mainly to the larger plasma surface area, 
steeper radial density gradients on the low-field side [1], and ELMing activity that expels particles almost exclusively through the low-field side [3]. As a result, particles that enter the SOL on the low-field side are the major source of fueling for both upper outer (baffle) target and the upper inner (dome) target. Although the particle source from the high-field side is lost when the magnetic balance changes from upper SN to DN, this loss would have only a minor impact on the total direct particle flow into the upper outer target (and hence the number of particles available for pumping). On the other hand, changing the magnetic balance from upper SN to DN severs the direct route of particles from the low-field side to the upper inner divertor, thus depriving it of the major source of particles available for pumping. Thus, one expects that changes in the magnetic balance near DN should have a greater effect on $\Gamma_{\mathrm{DOME}}$ than on $\Gamma_{\mathrm{BAF}}$.

The above discussion assumes that the number of particles exhausted by either pump is proportional to the number of particles striking the divertor targets. In turn, this assumes that neutrals that are "born" at the divertor target, are vectored toward a plenum entrance, and reach the plenum entrance without undergoing re-ionization, i.e., the mean free ionization pathlength of these neutrals $\left(\lambda_{\mathrm{ei}}\right)$ is greater than the distance $(D)$ from their origin to the cryopump entrance. Because the divertor separatrix strike points were adjacent to their respective plenum entrances and because $\lambda_{\mathrm{ei}}>D$, we expect that the fraction of the total number of particles striking the target that are pumped is largely a function of divertor geometry and the ion particle flux profile closest to the plenum entrance [11].

That pumping rate and recycling are linked by a near-linear relationship, as is shown in Figs. $(4 a, b)$, follows from the above discussion. The intensity of the recycling light near the openings to the dome and baffle plenums (i.e., $\Phi_{\mathrm{DOME}}$ and $\Phi_{\mathrm{BAF}}$, respectively) should be approximately 
proportional to the particle flux at the targets in front of their respective plenum entrances [12]. In turn, this leads to: $\Gamma_{\mathrm{DOME}} \propto \Phi_{\mathrm{DOME}}$ and $\Gamma_{\mathrm{BAF}} \propto \Phi_{\mathrm{BAF}}$.

Estimates of the pumping effectiveness depend on geometry (e.g., relative placement of the separatrix target to the plenum entrance) and the specifics of the pumping system design, and will not be addressed here. It is interesting, however, to speculate where active particle pumping might be most effective. For this, the poloidal locations of strong recycling for non-actively pumped discharges can provide insight, based on the above arguments. Figure 5 plots the ratios $\Phi_{\mathrm{DOME}-\mathrm{U}} / \Phi_{\mathrm{BAF}-\mathrm{U}}$ for similar-shaped but non-actively pumped discharges versus $d R s e p$, as reported previously [4]. Superimposed on Fig. 5 are the $\Gamma_{\mathrm{DOME}} / \Gamma_{\mathrm{BAF}}$ data from Fig. 2. The dependence of $\Phi_{\mathrm{DOME}-\mathrm{U}} / \Phi_{\mathrm{BAF}-\mathrm{U}}$ on $d R s e p$ is similar to that of $\Gamma_{\mathrm{DOME}} / \Gamma_{\mathrm{BAF}}$, and both $\Phi_{\mathrm{DOME}-\mathrm{U}} / \Phi_{\mathrm{BAF}-\mathrm{U}}$ and $\Gamma_{\mathrm{DOME}} / \Gamma_{\mathrm{BAF}}$ have similar responses to a reversal in the $B \times \nabla B$ ion drift direction. A further examination of this recycling data from non-actively pumped plasmas also showed that $\Phi_{\mathrm{BAF}-\mathrm{U}}$ was fairly insensitive to changes in dRsep near DN [4], as was $\Gamma_{\mathrm{BAF}}$, and that $\Phi_{\text {DOME-U }}$ was highly sensitive to changes in magnetic balance near DN [4], as was $\Gamma_{\text {DOME }} \cdot$

The above comparisons of $\Gamma_{\mathrm{DOME}} / \Gamma_{\mathrm{BAF}}$ with $\Phi_{\mathrm{DOME}-\mathrm{U}} / \Phi_{\mathrm{BAF}-\mathrm{U}}$ indicate that similar physics apply to both. While detailed modeling of the actively-pumped plasmas discussed in this paper is ongoing, previous modeling of the SOL and divertor of a non-actively pumped DN plasma using the 2-D fluid modeling edge transport code UEDGE [13] has shown that the observed asymmetries in $\Phi_{\mathrm{DOME}-\mathrm{U}} / \Phi_{\mathrm{BAF}-\mathrm{U}}$ (and, by extension, $\Gamma_{\mathrm{DOME}} / \Gamma_{\mathrm{BAF}}$ ) were consistent with the presence of $E \times B$ and $B \times \nabla B$ particle drifts. The electric field was mainly generated by the radial gradient in the electron temperature with respect to the flux surfaces in the private flux region (PFR) and its direction is always away from the X-point. For example, if 
the $B \times \nabla B$ ion drift direction was toward the upper divertor of the DN, UEDGE indicates that the $E \times B$ drift transports ions through the PFR from the outer plate to the inner plate in the upper divertor, ultimately resulting in an enhanced recycling at that inboard plate. This process was reversed in the lower divertor, as the $E \times B$ drift transports ions from the inner to the outer divertor through the PFR, ultimately resulting in a reduced recycling at that inboard plate. Details of the modeling assumptions are discussed in Ref. 4. The UEDGE qualitative predictions for $\Phi_{\text {DOME-U }} / \Phi_{\text {BAF-U }}$ in both upper and lower divertors are shown as open circles and open squares in Fig. 5, and are consistent with the experimental values of $\Phi_{\text {DOME-U }} / \Phi_{\mathrm{BAF}-\mathrm{U}}$,

These results show that particle removal at the divertor targets depends on both the magnetic balance and the direction of the $B \times \nabla B$ particle drift. Particle removal in the DN is particularly sensitive to changes in magnetic balance, and particle drifts in the SOL and divertor(s) may further redistribute particles between the inner and outer divertor target(s).

\section{Acknowledgment}

This work was supported in part by the U.S. Department of Energy under DE-FC0204ER54698, *W-7405-ENG-48, DE-AC05-00OR22725, and DE-AC04-94AL85000. 


\section{References}

[1] T.W. Petrie, C.M. Greenfield, R.J. Groebner, A.W. Hyatt, R.J. Lahaye, et al., J. Nucl. Mater. 290-293 (2001) 935.

[2] T.W. Petrie, M.E. Fenstermacher, C.J. Lasnier, Fusion Technol. 39 (2001) 916.

[3] T.W. Petrie, J.G. Watkins, L.L. Lao, P.B. Snyder, Nucl. Fusion 43 (2003) 910.

[4] T.W. Petrie, J.G. Watkins, L.R. Baylor, N.H. Brooks, M.E. Fenstermacher, et al., J. Nucl. Mater. 313-316 (2003) 834.

[5] A.V. Chankin, J. Nucl. Mater. 241-243 (1997) 199.

[6] J.A. Boedo, M.J. Schaeffer, R. Maingi, C.J. Lasnier, J.G. Watkins, Proc. of the $26^{\text {th }}$ Euro. Conf. on Control. Fusion and Plasma Physics, Maastricht, Vol. 23J, 1999 (European Physical Society) p. 1185.

[7] T.D. Rognlien, G.D. Porter, D.D. Ryutov, J. Nucl. Mater. 266-269 (1999) 654.

[8] M.E. Rensink, C.J. Lasnier, T.W. Petrie, G.D Porter, and T.D. Rognlien, Contrib. Plasma Phys. 42 (2002) 181.

[9] P. Gohil, M.A. Mahdavi, L.L. Lao, K.H. Burrell, M.S. Chu, et al., Phys. Rev. Lett. 61 (1988) 1603.

[10] P.N. Yushmanov, et al., Nucl. Fusion 30 (1990) 1999.

[11] R. Maingi, J.G. Watkins, M.A. Mahdavi, and L.W. Owen, "Pump Plenum Pressure Dependence on Divertor Plasma Parameters and Magnetic Geometry in the DIII-D Tokamak,” General Atomics Report GA-G23057 (1999).

[12] P.C. Stangeby, The Plasma Boundary of Magnetic Fusion Devices, Institute of Plasma Physics Publishing Ltd, 2000, Chapter 5.

[13] T.D. Rognlien, et al., Plasma Phys. 34 (1994) 362. 


\section{Captions}

Fig. 1. The three basic equilibria used in this study: (a) DN biased toward the upper divertor $($ dRsep $=+1.2 \mathrm{~cm}),(\mathrm{b})$ balanced DN $($ dRsep $=0)$, and (c) DN biased toward the lower divertor $($ dRsep $=-1.2 \mathrm{~cm})$. The "dome" and "baffle" pumps exhaust particles at the upper inner leg divertor target and upper outer leg divertor target, respectively. The "primary" separatrix is shown as a heavy line; the secondary separatrix is shown as a lighter line. Characteristic parameters: $I_{\mathrm{p}}=1.2 \mathrm{MA},\left|B_{\mathrm{T}}\right|=1.85 \mathrm{~T}$, and $P_{\mathrm{INJ}}=6-11 \mathrm{MW}$.

Fig.2. The ratio $\Gamma_{\mathrm{DOME}} / \Gamma_{\mathrm{BAF}}$ depends on $d$ Rsep for cases where the $B \times \nabla B$ direction is toward the upper divertor (solid circles) and toward the lower divertor (solid squares).

Fig. 3. $\Gamma_{\mathrm{DOME}}$ changed much more than $\Gamma_{\mathrm{BAF}}$ between $d R$ sep $=+1$ to $d R s e p=-1 \mathrm{~cm}$. The values of $\Gamma_{\mathrm{INJ}}, \Gamma_{\mathrm{DOME}}, \Gamma_{\mathrm{BAF}}$, and $d N_{\mathrm{c}} / d t$ were determined by measurement, while $\Gamma_{\mathrm{WALL}}$ was deduced from Eq. (1). The data were characterized by $n_{\mathrm{e}, \mathrm{PED}}=$ $(0.37-0.40) \times 10^{20}, \Gamma_{\mathrm{INJ}}=(11-17)$ torr liter/s. The direction of the $B \times \nabla B$ ion particle drift was toward the upper divertor.

Fig. 4. (a) $\Gamma_{\mathrm{DOME}}$ and (b) $\Gamma_{\mathrm{BAF}}$ are plotted as a function of the recycling radiation adjacent to the opening to their respective dome and baffle plenums. 
Fig. 5. Polynomial fits to $\Gamma_{\mathrm{DOME}} / \Gamma_{\mathrm{BAF}}$ for $B \times \nabla B$ directed toward the upper divertor (solid curve) and for $B \times \nabla B$ directed away from the upper divertor (dashed curve) are plotted versus dRsep for the actively pumped plasmas in Fig. 2. Superimposed are the recycling ratios $\Phi_{\text {DOME-U }} / \Phi_{\mathrm{BAF}-\mathrm{U}}$ for $B \times \nabla B$ directed toward the upper divertor (solid circles) and for $B \times \nabla B$ directed away from the upper divertor (solid boxes) for similar but non-actively pumped plasmas. The UEDGE-generated recycling ratios at each divertor is modeled for a non-actively pumped DN case, assuming the $B \times \nabla B$ ion drift direction is toward the upper divertor; the prediction for the "upper divertor" is shown as an open circle and for the "lower divertor" as an open box. 


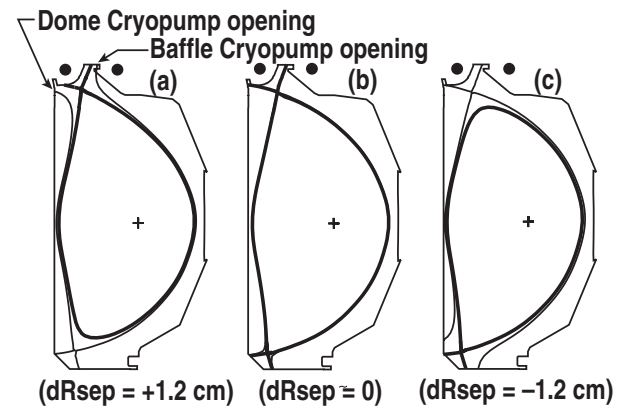

T.W. Petrie Figure 1 


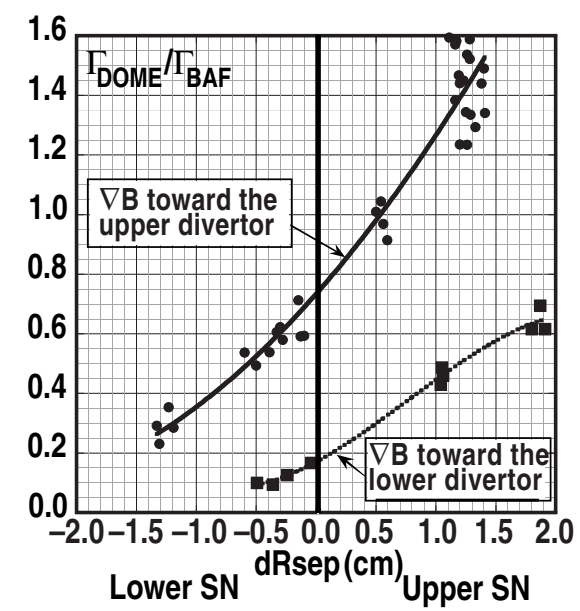

T.W. Petrie Figure 2 


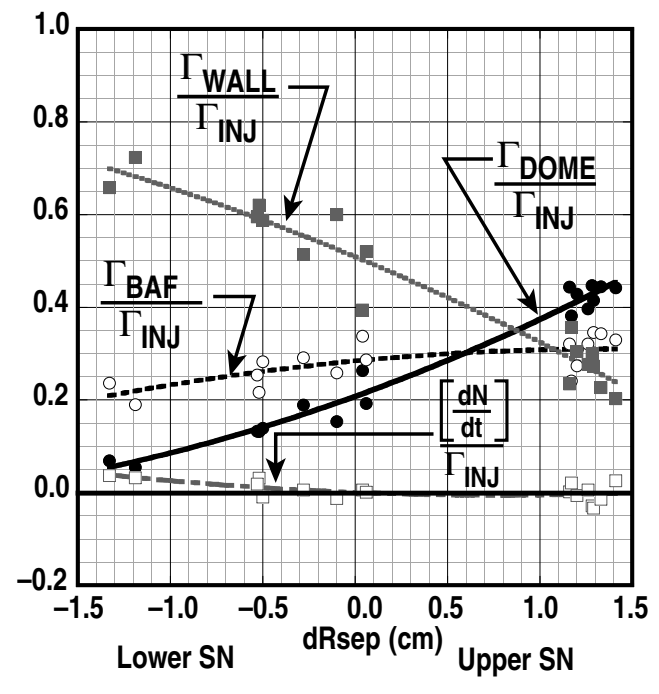

T.W. Petrie Figure 3 

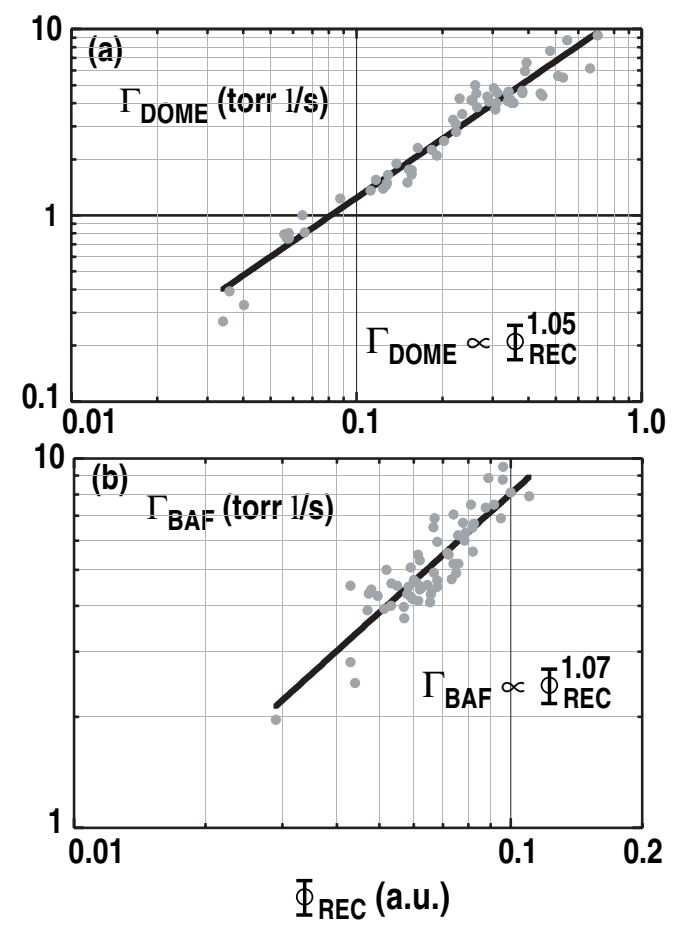

T.W. Petrie Figure 4 


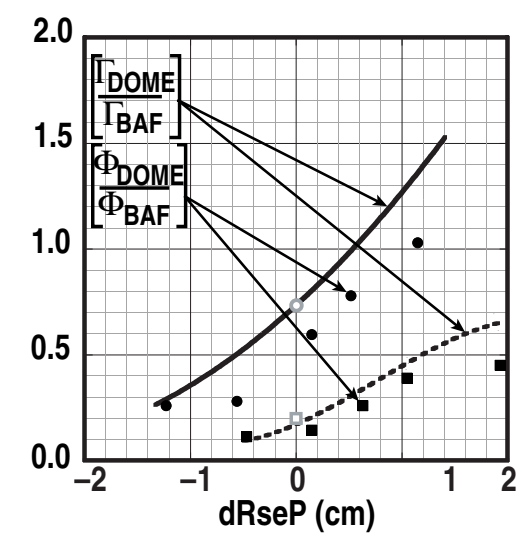

T.W. Petrie Figure 5 